\title{
Is [18F]-2-fluoro-2-deoxy-D-glucose (FDG) scintigraphy with non-dedicated positron emission tomography useful in the diagnostic management of suspected metastatic thyroid carcinoma in patients with no detectable radioiodine uptake?
}

Francesco Giammarile, Zakia Hafdi ${ }^{1}$, Claire Bournaud ${ }^{2}$, Marc Janier ${ }^{3}$, Claire Houzard ${ }^{1}$, Claude Desuzinges, Roland $\mathrm{Itti}^{1}$, Geneviève Sassolas ${ }^{1}$ and Françoise Borson-Chazot ${ }^{1}$

Nuclear Medicine Department, Centre Léon Bérard, 28, rue Laennec, 69373 Lyon cedex 08, France, ${ }^{1}$ Nuclear Medicine Department, Hôpital Neuro-cardiologique, Lyon, France, ${ }^{2}$ Department of Endocrinology, Hôpital Lyon-Sud, France and ${ }^{3}$ Nuclear Medicine Department, Hôpital Edouard Herriot and CERMEP, Lyon, France

(Correspondence should be addressed to F Giammarile; Email: giammari@lyon.fnclcc.fr)

\begin{abstract}
Objective: Dedifferentiation of thyroid cancer leads to an inability of thyroid cells to concentrate iodine. In these cases, imaging methods that allow an accurate detection of recurrence and/or metastases at an early stage are essential for an adequate management of patients. Positron emission tomography using [18F]-2-fluoro-2-deoxy-D-glucose and a dedicated (dPET-FDG) or non-dedicated (nPET-FDG) camera has been suggested as a potential tool for the detection of tumour foci.

Design and methods: This prospective study was undertaken to evaluate nPET-FDG in 51 consecutive patients (18 men, 33 women) with differentiated thyroid cancer ( 33 papillary, 11 follicular, four insular and three oncocytic (Hürthle-cell) thyroid carcinomas). Selection criteria were high thyroglobulin (Tg) levels (>10 ng/ml off-levothyroxine treatment) and no detectable radioiodine uptake, on a whole body scan performed with a high dose, in the absence of iodine contamination.

Results: Results were interpreted in terms of assumed presence of tumoral tissue. Sensitivity of nPETFDG was similar to that of conventional imaging modalities $(67 \%)$. False negative nPET-FDG $(n=16)$ were observed mostly in cases of micro-lesions (lymph nodes or lung metastases). Conversely, nPETFDG identified new tumoral sites in 11 cases. Better sensitivity was found for nPET-FDG in patients with Tg levels higher than $15 \mu \mathrm{g} / \mathrm{l}(P<0.05)$. On a patient basis, results of nPET-FDG were equivalent to that of dPET-FDG. Finally, nPET-FDG changed treatment strategy in seven patients.

Conclusions: nPET-FDG has a high sensitivity for the detection of tumour sites in patients when pathological iodine uptake cannot be demonstrated and appears to be a useful method in patients with elevated Tg levels, especially when dedicated PET is either unavailable or impractical.
\end{abstract}

European Journal of Endocrinology 149 293-300

\section{Introduction}

The follow-up of patients with differentiated thyroid cancer, after total thyroidectomy and radioiodine ablation therapy, is based on the combination of human thyroglobulin $(\mathrm{Tg})$ serum monitoring and radioiodine whole body scans (WBS) (1). When tumoral sites are able to concentrate radioiodine, 131-iodine $\left({ }^{131} \mathrm{I}\right)$ therapy is effective. However, in a third of patients with elevated Tg levels, which indicate persistent or recurrent disease, there is no identifiable focus on the WBS, even when performed after a therapeutic administration of ${ }^{131} \mathrm{I}(2)$. It is generally considered that the lack or the loss over time of the ability of tumoral cells to trap iodine (while still retaining the ability to secrete $\mathrm{Tg}$ ) may be associated with poorly differentiated tumours and indicate aggressive behaviour and poor prognosis $(3,4)$. In such patients, who cannot benefit from iodine therapy, precise localisation of tumoral sites is essential to define the therapeutic strategy, namely, a surgical treatment whenever possible, or palliative measures.

Conventional imaging procedures (CIP), mostly ultrasound of neck and abdomen, computerised tomography (CT) and/or magnetic resonance imaging (MRI), and bone scintigraphy, are effective but poorly 
specific since it is difficult to distinguish active disease from fibrotic tissue, particularly when normal anatomy is altered by previous treatments. The use of CT contrast enhancement imaging is restricted if planned less than 6 weeks before a further radioiodine administration.

Functional imaging modalities offer the possibility for a whole body evaluation. Several radiopharmaceutics such as thallium-201 chloride, technetium-99m labelled isonitrile compounds (sestamibi or tetrafosmin) and [indium-111 DTPA D Phe]octreotide have been therefore proposed as a potential tool for the diagnosis of recurrences in thyroid carcinomas, but results have proved controversial with sensitivities depending on patient selection. In general, all these techniques are viable in the presence of a sizeable tumour but, having poor sensitivities, they are incapable of detecting micrometastases $(2,5-8)$.

Positron emission tomography (PET) using [18F]-2fluoro-2-deoxy-D-glucose (FDG), an analogue of glucose, has been widely used in clinical oncology (9). In thyroid cancer, previous studies have pointed to the high sensitivity of the examination for the detection of recurrences, especially in patients whose tumours do not concentrate iodine. For instance, Grunwald et al. (10) reported in such patients a sensitivity of $85 \%$ and in the study conducted by Helal et al., PETFDG was significantly more sensitive than CIP in localising tumoral sites (11). These results may be explained by a higher glucose metabolism in these tumours, often poorly differentiated $(12-15)$, and PET-FDG has been suggested as a prognostic indicator $(16-18)$. Most of the above data have been obtained with dedicated cameras (dPET). An alternative method for FDG detection is the use of a gamma camera with coincidence detection capability, called non-dedicated PET (nPET) (19, 20). The sensitivity and clinical impact of this easily available and less expensive method have not yet been precisely evaluated in thyroid cancer.

The aim of this study, performed in a prospective series of 51 patients with elevated $\mathrm{Tg}$ and negative WBS without any iodine contamination, was to evaluate nPET by reference to CIP and clinical follow-up, and to compare the performances of nPET and dPET.

\section{Materials and methods}

\section{Patients}

Fifty-one patients with differentiated thyroid cancer (18 men, 33 women; age range: $14-80$ years; mean age: 54 years) were studied prospectively. Total thyroidectomy had been performed 1-34 years previously in all cases. The histopathological analysis revealed 33 papillary, 11 follicular, four insular and three oncocytic (Hürthlecell) thyroid carcinomas. The disease stage at diagnosis was defined according to the National Thyroid Cancer
Treatment Cooperative Study staging system (21). Surgical treatment was followed by ${ }^{131}$ I ablative therapy with 1.9-3.7 GBq (depending on the radioiodine uptake in the thyroid bed). Subsequently, the patients received one to nine therapeutic doses of ${ }^{131} \mathrm{I}$ (cumulative range: $1.9-48.5 \mathrm{GBq}$, average dose: $12.4 \mathrm{GBq}$ ) because of elevated serum thyroglobulin levels (mean $771 \pm 1618 \mu \mathrm{g} / \mathrm{l}$, range: $21-10893 \mu \mathrm{g} / \mathrm{l})$. In eight patients, the WBS, performed after the therapeutic dose of radioiodine, showed no uptake at all. In the other 43 patients, repeated ${ }^{131}$ I treatments resulted in the disappearance of radioiodine uptake in tumour sites. However, the $\mathrm{Tg}$ level remained mostly elevated (mean 644 $1966 \mu \mathrm{g} / \mathrm{l}$, range: $0.7-11617 \mu \mathrm{g} / \mathrm{l}$, during levothyroxine treatment), with no anti-Tg antibody. In 17 patients, the elevated $\mathrm{Tg}$ level was the only evidence of the presence of tumour at the time of FDG exploration. The other 34 patients had been treated for metastases visualized by CIP. The mean delay between the last radioiodine treatment and FDG scintigraphy was $21 \pm 28$ months (range: 1 week to 10 years).

The study was performed according to the Helsinki Guidelines and was approved by the local ethics committee. Each patient gave informed written consent.

\section{Conventional imaging methods}

A WBS was performed 3-4 days after the administration of ${ }^{131} \mathrm{I}(1.9-5.6 \mathrm{GBq})$, under endogenous thyrotrophin (TSH) stimulation (TSH level higher than $30 \mathrm{mU} / \mathrm{l})$, using a dual-head large-field-of-view gamma camera equipped with high-energy collimators.

Serum Tg and anti-Tg antibody were measured by IRMA (PASTEUR) and recorded at the time of WBS and on the day of nPET-FDG scintigraphy.

CIP first included chest X-rays and neck ultrasound. Spiral CT with contrast enhancement and/or MRI of the neck and chest (and occasionally the abdomen) were performed by two experienced radiologists, mostly when the two first examinations were negative or doubtful, and always in the case of unexpected FDG findings. A technetium-99m diphosphonate scan was performed in cases of clinically suspected bone metastases.

\section{PET-FDG acquisitions (Fig. 1)}

Examinations were performed under levothyroxine suppression therapy (TSH $<0.1 \mathrm{UI}$ ), except in six cases (Table 1). Images were generally obtained in fasting patients $60 \mathrm{~min}$ after intravenous administration of 2 (for nPET) or 4-5 (for dPET) MBq/kg of FDG. Dedicated PET-FDG was performed the same day in seven patients or shortly after nPET (less than 15 days) in 13 patients. When the two examinations were planned for the same day, nPET-FDG images were obtained $2-3 \mathrm{~h}$ after 
Table 1 Patient characteristics and results.

\begin{tabular}{|c|c|c|c|c|c|c|c|c|}
\hline Pt & G & A & $\mathbf{H}$ & $\mathrm{Tg}$ & nPET & CIP & $V f$ & $\mathrm{Tm}$ \\
\hline 1 & $M$ & 48 & 1 & 12.5 & 0 cer $(\mathrm{FN})$ & 3 cer (TP) & B & Survey \\
\hline 2 & $\mathrm{~F}$ & 45 & $\mathrm{P}$ & 9 & 1 cer (TP) & 0 cer (FN) & $\mathrm{H}$ & Surgery \\
\hline 3 & $\mathrm{~F}$ & 24 & $\mathrm{P}$ & 5.3 & 2 cer (TP) & 2 cer (TP) & $\mathrm{H}$ & Surgery \\
\hline 4 & $\mathrm{~F}$ & 23 & $\mathrm{P}$ & 3 & 1 cer (TP) & $1 \operatorname{cer}(\mathrm{TP})$ & $\mathrm{H}$ & Surgery \\
\hline 5 & $\mathrm{~F}$ & 22 & $\mathrm{P}$ & 7.4 & $0(\mathrm{FN})$ & $0(\mathrm{FN})$ & $\mathrm{F}$ & Survey \\
\hline 6 & $\mathrm{~F}$ & 24 & $\mathrm{P}$ & 0.7 & 0 cer (FN) & $4 \operatorname{cer}(\mathrm{TP})$ & $\mathrm{H}$ & Surgery \\
\hline 7 & $M$ & 29 & $\mathrm{P}$ & 6 & $0(\mathrm{FN})$ & $0(\mathrm{FN})$ & $\mathrm{F}$ & Survey \\
\hline 8 & $\mathrm{~F}$ & 31 & $P$ & 13.4 & 0 cer (FN) & 1 cer (TP) & B & Survey \\
\hline 9 & $M$ & 45 & $\mathrm{P}$ & 13.6 & 1 cer (TP) & 1 cer (TP) & $\mathrm{H}$ & Surgery \\
\hline 10 & $\mathrm{~F}$ & 29 & $\mathrm{P}$ & 1.6 & 0 cer (FN) & $2 \operatorname{cer}(\mathrm{TP})$ & B & Survey \\
\hline \multirow[t]{2}{*}{11} & $M$ & 67 & $\mathrm{P}$ & 2.4 & $1 \operatorname{cer}(\mathrm{TP})$ & 1 cer (TP) & $\mathrm{B}, \mathrm{F}$ & Survey \\
\hline & & & & & 1 pulm (TP) 1 med (TP) & 0 pulm (FN) 1 med (TP) & & \\
\hline \multirow[t]{2}{*}{12} & $\mathrm{~F}$ & 67 & $\mathrm{~F}$ & 12 & $1 \operatorname{cer}(\mathrm{TP})$ & $0 \operatorname{cer}(\mathrm{FN})$ & $\mathrm{B}, \mathrm{F}$ & Redifferentiation \\
\hline & & & & & 3 pulm (TP) & 2 pulm (TP) & & \\
\hline 13 & $\mathrm{~F}$ & 27 & $\mathrm{P}$ & 1.4 & 1 bone (TP?) & 0 bone (FN?) & $\mathrm{F}$ & Survey \\
\hline 14 & $\mathrm{~F}$ & 65 & $\mathrm{~F}$ pd & 11.2 & $0(\mathrm{FN})$ & $0(\mathrm{FN})$ & $\mathrm{F}$ & Survey \\
\hline \multirow[t]{2}{*}{15} & $M$ & 52 & 1 & 7 & $0 \operatorname{cer}(\mathrm{FN})$ & 1 cer (TP) & $\mathrm{B}, \mathrm{F}$ & Radiotherapy \\
\hline & & & & & 1 med (TP) & 1 med (TP) & & \\
\hline 16 & $M$ & 50 & $\mathrm{P}$ & 2.1 & $0(\mathrm{FN})$ & $0(\mathrm{FN})$ & $\mathrm{F}$ & Survey \\
\hline 17 & $\mathrm{~F}$ & 10 & $\mathrm{P}$ & 10 & 1 cer (TP) & 1 cer (TP) & $\mathrm{H}$ & Surgery \\
\hline 18 & $M$ & 21 & $\mathrm{P}$ & 2.1 & 4 cer (TP) & 2 cer (TP) & $\mathrm{H}$ & Surgery \\
\hline 19 & $\mathrm{~F}$ & 11 & $\mathrm{P}$ & 33 & $0(\mathrm{FN})$ & $0(\mathrm{FN})$ & $\mathrm{F}$ & Survey \\
\hline 20 & $\mathrm{~F}$ & 54 & $\mathrm{P}$ & 36 & $0(\mathrm{FN})$ & $0(\mathrm{FN})$ & $\mathrm{F}$ & Survey \\
\hline 21 & $M$ & 66 & $\mathrm{P}$ & 38 & 1 cer (TP) & 1 cer (TP) & $\mathrm{H}$ & Surgery \\
\hline 22 & $\mathrm{~F}$ & 54 & $\mathrm{P}$ & 19.1 & $0(\mathrm{FN})$ & $0(\mathrm{FN})$ & $\mathrm{F}$ & Survey \\
\hline $23^{*}$ & $\mathrm{~F}$ & 43 & $\mathrm{~F}$ pd & 38 & 1 pulm (TP) & 1 pulm (TP) & $\mathrm{H}$ & Surgery \\
\hline 24 & $\mathrm{~F}$ & 67 & $\mathrm{O}$ & 23.1 & $1 \operatorname{cer}(\mathrm{TP})$ & $1 \operatorname{cer}(\mathrm{TP})$ & $\mathrm{H}$ & Surgery \\
\hline 25 & $\mathrm{~F}$ & 35 & $\mathrm{P}$ & 50 & 0 pulm (FN?) & 1 pulm (TP?) & $\mathrm{F}$ & Survey \\
\hline 26 & $M$ & 10 & $\mathrm{P}$ & 49 & $1 \operatorname{cer}(\mathrm{TP})$ & $1 \operatorname{cer}(\mathrm{TP})$ & $\mathrm{H}$ & Surgery \\
\hline 27 & $\mathrm{~F}$ & 51 & $\mathrm{~F} \mathrm{pd}$ & 17 & 1 cer (TP) $0 \mu$ pulm (FN) & 0 cer (FN) $1 \mu$ pulm (TP) & $\mathrm{H}, \mathrm{F}$ & Surgery \\
\hline 28 & $\mathrm{~F}$ & 45 & $\mathrm{O}$ & 96 & 1 pulm (TP) 1 med (TP) & 1 pulm (TP) 1 med (TP) & $\mathrm{H}$ & Surgery \\
\hline 29 & $M$ & 50 & $\mathrm{P}$ & 29 & 0 upulm (FN) & 3 upulm (TP) & $\mathrm{F}$ & Survey \\
\hline $30^{*}$ & $\mathrm{~F}$ & 42 & $\mathrm{~F}$ & 130 & $4 \operatorname{cer}(\mathrm{TP})$ & $4 \operatorname{cer}(\mathrm{TP})$ & $\mathrm{H}$ & Surgery \\
\hline $31^{*}$ & $M$ & 31 & $\mathrm{P}$ & 90 & 1 cer (TP) & 1 cer (TP) & $\mathrm{H}$ & Surgery \\
\hline 32 & $\mathrm{~F}$ & 63 & I & 59 & 1 med (TP) & 0 med (FN) & $\mathrm{H}$ & Surgery \\
\hline \multirow[t]{2}{*}{33} & $\mathrm{~F}$ & 66 & $\mathrm{P}$ & 43.5 & $1 \operatorname{cer}(\mathrm{TP})$ & $0 \operatorname{cer}(\mathrm{FN})$ & $\mathrm{H}, \mathrm{F}$ & Redifferentiation \\
\hline & & & & & 0 pulm (FN) 0 med (FN) & 1 pulm (TP) 1 med (TP) & & \\
\hline 34 & $\mathrm{~F}$ & 38 & $\mathrm{P}$ & 245 & 4 pulm (TP) 1 med (TP) & 4 pulm (TP) 1 med (TP) & $\mathrm{F}$ & Redifferentiation \\
\hline $35^{\star}$ & $\mathrm{F}$ & 18 & $\mathrm{P}$ & 35 & $1 \operatorname{cer}(\mathrm{TP})$ & $1 \operatorname{cer}(\mathrm{TP})$ & $\mathrm{H}$ & Surgery \\
\hline 36 & $\mathrm{~F}$ & 63 & 0 & 142 & $0(\mathrm{FN})$ & $0(\mathrm{FN})$ & $\mathrm{F}$ & Survey \\
\hline \multirow[t]{2}{*}{37} & $\mathrm{~F}$ & 58 & $\mathrm{~F} \mathrm{pd}$ & 1755 & M pulm (TP) 1 med (TP) & M pulm (TP) 1 med (TP) & $\mathrm{F}$ & Redifferentiation \\
\hline & & & & & 0 hep $(\mathrm{FN}) \dagger$ & 1 hep (TP) & & \\
\hline $38^{*}$ & $\mathrm{~F}$ & 50 & $\mathrm{P}$ & 168 & M pulm (TP) 1 bone (TP) & 0 pulm (FN) 0 bone (FN) & $\mathrm{F}$ & Redifferentiation \\
\hline $39 *$ & $M$ & 72 & $\mathrm{P}$ & 579 & 2 cer (TP) 2 pulm (TP) & 1 cer (TP) M pulm (TP) & $\mathrm{H}$ & Surgery \\
\hline \multirow[t]{2}{*}{40} & M & 74 & $\mathrm{P}$ & 161 & 1 cer (TP) M axil (TP) & 1 cer (TP) M axil (TP) & $\mathrm{B}, \mathrm{F}$ & Radiotherapy \\
\hline & & & & & 1 bone (TP?) & 0 bone (FN?) & & \\
\hline 41 & $\mathrm{~F}$ & 62 & $\mathrm{~F}$ & 5250 & 1 med $(\mathrm{TP})$ & 1 med $(T P)$ & $\mathrm{H}$ & Surgery/Radiotherapy \\
\hline \multirow[t]{3}{*}{$42^{*}$} & $\mathrm{~F}$ & 69 & $\mathrm{P}$ & 11617 & $4 \operatorname{cer}(\mathrm{TP}) 1 \mathrm{med}(\mathrm{TP})$ & $4 \operatorname{cer}(\mathrm{TP}) 0$ med (FN) & $\mathrm{F}$ & Redifferentiation \\
\hline & & & & & 1 bone (TP) 1 abd (TP) & 1 bone (TP) +1 bone (FP) & & \\
\hline & & & & & & 0 abd (FN) & & \\
\hline 43 & M & 60 & $\mathrm{~F}$ & 850 & $0(\mathrm{FN})$ & $0(\mathrm{FN})$ & $\mathrm{F}$ & Survey \\
\hline $44^{*}$ & M & 71 & $\mathrm{~F}$ pd & 638 & 1 cer $(\mathrm{TP})$ & 0 cer $(\mathrm{FN})$ & $\mathrm{H}$ & Surgery \\
\hline 45 & $\mathrm{~F}$ & 29 & $\mathrm{~F}$ & 187 & $\mathrm{M}$ cer (TP) 1 med (TP) & 2 cer (TP) 0 med (FN) & $\mathrm{H}$ & Surgery \\
\hline 46 & $M$ & 70 & $\mathrm{~F} \mathrm{pd}$ & 1964 & 1 bone (TP) & 0 bone (FN) & $\mathrm{F}$ & Radiotherapy \\
\hline 47 & $\mathrm{~F}$ & 53 & $P$ & 609 & 1 bone (TP) & 1 bone (TP) & $\mathrm{F}$ & Radiotherapy \\
\hline 48 & M & 49 & I & 210 & 0 pulm (FN) 2 bone (TP) & M pulm (TP) 1 bone (TP) & $\mathrm{F}$ & Redifferentiation \\
\hline 49 & $\mathrm{~F}$ & 53 & $\mathrm{P}$ & 5850 & 1 med (TP) & 2 med (TP) & $\mathrm{H}$ & Surgery \\
\hline 50 & $M$ & 62 & $\mathrm{P}$ & 278 & M pulm (TP) 3 bone (TP?) & M pulm (TP) 0 bone (FN?) & $\mathrm{F}$ & Redifferentiation \\
\hline 51 & $\mathrm{~F}$ & 41 & $\mathrm{P}$ & 171 & 1 abd (FP: bladder) & $0(\mathrm{FN})$ & $\mathrm{F}$ & Survey \\
\hline
\end{tabular}

Pt: Patient number; *patients with no evidence of disease on initial WBS.

G: Gender (male or female).

A: Age at diagnosis (in years).

$\mathrm{H}$ : Histology: $\mathrm{P}$, papillary; $\mathrm{F}$, follicular, I, insular; and $\mathrm{O}$, oncocytic thyroid carcinomas pd, poorly differentiated forms.

$\mathrm{Tg}$ : $\mathrm{Tg}$ the day of FDG examination (in $\mu \mathrm{g} / \mathrm{l}$ ) (all patients had undetectable TSH levels except nos $35,48,49,50$ and 51 ).

nPET: Non-dedicated PET using FDG results. TP, true positive; FN, false negative, FP, false positive. abd, abdominal; cer, cervical; hep, hepatic; M axil, Multiple axillary; med, mediastinal; pulm, pulmonary; M pulm, Multiple pulmonary; $\mu$ pulm, Micro-metastasis pulmonary. †One hepatic lesion seen only on dPET.

CIP: TP, true positive; FN, false negative; FP, false positive.

Vf: verification by cytology $(B)$, histology $(\mathrm{H})$ or follow-up $(\mathrm{F})$.

$\mathrm{Tm}$ : Therapeutic management (in italics: nPET-FDG findings changed management). 
the initial injection of FDG for dPET, in order to avoid tracer re-injection.

nPET-FDG images were acquired using a three-head large field-of-view gamma camera equipped with coincidence detection of $511 \mathrm{keV}$ annihilation quanta (IRIX Philips Medical Systems, Cleveland, OH, USA) in all the patients. Three acquisitions of neck, chest and abdomen involved a $120^{\circ}$ rotation of each detector, with 60 steps of $20-25 \mathrm{~s}$ and the total scanning time was approximately $1.5 \mathrm{~h}$. The non-attenuated corrected images were reconstructed using the iterative reconstruction technique.

dPET-FDG images were acquired using a PET camera, with an axial field of view of $15.2 \mathrm{~cm}$ (ECAT EXACT $\mathrm{HR}+$ Siemens/CTI, Knoxville, TN, USA) in 20 patients, including all the cases showing no pathological uptake on nPET-FDG. All scannings were performed in a threedimensional (septa-out) mode, from the cerebellum to the femur, and the total scanning time was approximately $1 \mathrm{~h}$. Transmission images using a 68Ge source were acquired for attenuation correction. Images were reconstructed using the OSEM technique (iterative reconstruction algorithm).

\section{Image interpretations and evaluation}

All scintigraphic images were analysed visually by at least two experienced nuclear physicians blinded to the clinical situation and the consensus was reported in the clinical record. All foci of increased abnormal uptake were interpreted as pathological sites (true or false positives). The regions of uptake were divided into five areas: neck, lung, mediastinum, bone and other (including the liver). Lesions were arbitrarily classified as multiple if more than four sites were detected. Overall, a positive examination in a patient was defined by at least one abnormal uptake in one site and results were expressed both in terms of positive patients and tumour sites. The scintigraphic results were compared retrospectively with conventional imaging studies, obtained within 6 months. If necessary, the CIP examinations were repeated with the radiologist aware of PET-FDG findings.

Since no single imaging modality is recognised as a gold standard to indicate the presence or absence of thyroid cancer, the evaluation criteria were defined in terms of assumed presence of tumoral tissue (because of persistent Tg levels) and not on the basis of CIP findings. Verification of a true positive finding was then achieved by positive cytology (performed by ultrasonography-guided fine-needle aspiration biopsy) or histology (performed after surgery) in 30 patients. In the other 21 patients, the final classification was based on the clinical outcome and biological follow-up, together with all imaging findings. Modifications of therapeutic management after FDG scintigraphy were recorded in all patients.

\section{Statistical analysis}

Lesion detectability in CIP, nPET and dPET-FDG are expressed as the percentage of patients with positive rates and compared using the $\chi^{2}$ test. The threshold of significance was set at 0.05 .

\section{Results}

\section{Overall results (Table 1)}

In nine patients, no tumour site could be detected with any of the available methods ('all negative patients'). Misleading findings occurred in patient no. 51, where nPET-FDG disclosed one false positive due to false interpretation of urinary activity in bladder. Two patients had equivocal findings: one patient had a bone lesion seen on nPET-FDG (patient no. 13) and one patient had a pulmonary metastasis seen on CIP (patient no. 25). In both cases, no confirmation of the presence of disease activity could be obtained.

The remaining 39 patients included in the study presented with unequivocal tumour sites (distant metastases, local recurrence or residual thyroid tumour), diagnosed by at least one imaging modality, and then confirmed either directly (histopathologically) or indirectly (by clinical follow-up).

Both CIP and nPET-FDG were unequivocally positive in 34 of $51(67 \%)$ patients, and were concordant in 29 positive cases $(85 \%)$. Thus, CIP in five patients and nPET in five other patients were the only diagnostic methods showing unequivocal pathological localisation. Dedicated PET was negative in the 16 patients showing no pathological uptake on nPET-FDG. In the remaining four patients who underwent both FDG scintigraphies, dPET results were similarly positive on nPET in three patients and more informative in only one (no. 37 ), with the identification of more lung metastases and of a liver site that were subsequently confirmed by CIP.

\section{Sensitivity according to tumour site (Table 2)}

In the 39 patients in whom a lesion was unequivocally detected, by combining the results of all the image modalities, the following tumour sites were detected: cervical (26 patients), mediastinal (11 patients), pulmonary (13 patients, including two diffuse micrometastases), skeletal (five patients); and other (three patients).

In these patients, nPET-FDG and CIP findings were comparable for neck recurrences, nPET-FDG was superior to CIP for mediastinal and bone localisation, and CIP was superior for lung localisation, mainly for miliary forms.

Overall, nPET-FDG identified new tumoral sites in 13 sites (five cervical, three mediastinal, two pulmonary, two skeletal and one abdominal) in 11 patients. 
Table 2 Site sensitivity.

\begin{tabular}{|c|c|c|c|c|c|c|c|c|}
\hline \multirow[b]{2}{*}{ Site } & \multicolumn{4}{|c|}{ CIP } & \multicolumn{4}{|c|}{ nPET } \\
\hline & TP & FN & $\mathrm{Se}$ & $\mathrm{Eq}$ & TP & $\mathrm{FN}$ & $\mathrm{Se}$ & $\mathrm{Eq}$ \\
\hline Cervical & 22 & 4 & 85 & - & 21 & 5 & 81 & - \\
\hline Mediastinal & 8 & 3 & 73 & - & 10 & 1 & 91 & - \\
\hline Pulmonary & $11(2 \mu)$ & 2 & 85 & $1 \mathrm{TP} ?$ & 9 & $4(2 \mu)$ & 69 & $1 \mathrm{FN}$ ? \\
\hline Bone & 3 & 2 & 60 & $3 \mathrm{FN}$ ? & 5 & 0 & 100 & 3 TP? \\
\hline Other & 2 & 1 & 67 & - & 2 & $1 *$ & 100 & - \\
\hline Total & 46 & 12 & 79 & - & 48 & 10 & 83 & - \\
\hline $\mathrm{FP}$ & \multicolumn{4}{|c|}{1 (irradiated bone) } & \multicolumn{4}{|c|}{1 (bladder) } \\
\hline
\end{tabular}

nPET, Non-dedicated PET using FDG results; TP, true positive; FN, false negative; FP, false positive; Se, sensitivity (\%); Eq: equivocal findings.

*One hepatic lesion seen only on PET. $\mu$, lesion size $<1 \mathrm{~cm}$.

For the five cervical sites, neck ultrasound was previously considered as non-informative. However, the examination found the lesions (subsequently confirmed by histopathology) when it was oriented by nPET-FDG results. Conversely, nPET-FDG was falsely negative in 11 tumoral sites (five cervical identified by neck ultrasound, one mediastinal, four pulmonary, and one skeletal) in 10 patients.

An irradiated bone was interpreted as a bone metastasis with bone scintigraphy and was negative with nPET-FDG (patient no. 42). The clinical follow-up confirmed nPET-FDG interpretation. The smallest lesion detected with nPET-FDG was a cervical metastasis of $8 \mathrm{~mm}$ diameter (patient no. 4).

\section{Scintigraphic sensitivity according to $\mathrm{Tg}$ (Table 3)}

To evaluate the influence of the Tg level on the sensitivity, the patients were divided into three groups, based on serum Tg levels obtained during levothyroxine therapy: less than $15 \mu \mathrm{g} / \mathrm{l}$ (group I), between 15 and $150 \mu \mathrm{g} / \mathrm{l}$ (group II), and higher than $150 \mu \mathrm{g} / \mathrm{l}$ (group III). Considering only the 45 patients who underwent scintigraphic examinations while the TSH level was undetectable, in group I nPET-FDG was unequivocally positive in nine of 18 patients $(50 \%)$, which is significantly lower than in the two other groups $(P<0.05$ for group I vs II + III). The lowest $\mathrm{Tg}$ with positive nPET-FDG findings was $2.1 \mu \mathrm{g} / \mathrm{l}$ (patient no. 18) and the highest $\mathrm{Tg}$ level with negative FDG scintigraphy was $3700 \mu \mathrm{g} / \mathrm{l}$ (patient no. 43).
Table 3 Scintigraphic sensitivity according to $\mathrm{Tg}$ in patients with undetectable TSH levels.

\begin{tabular}{lcccc}
\hline & \multicolumn{4}{c}{ Tg level $(\mu \mathrm{g} / \mathrm{l})$} \\
\cline { 2 - 5 } mPET & $\mathrm{Tg}<15$ & $15>\mathrm{Tg}<150$ & $\mathrm{Tg}>150$ & Total \\
\hline Positive & 9 & 11 & 10 & 30 \\
Total & 18 & 16 & 11 & 45 \\
Positive rate (\%) & 50 & 69 & 91 & 67 \\
Equivocal and ‘all & 6 & 4 & 1 & 11 \\
$\quad$ negative' patients & & & & \\
\hline
\end{tabular}

\section{Scintigraphic sensitivity according to histology and stage (Table 4)}

In the 33 patients with papillary carcinoma, nPET findings were unequivocally positive in 20 patients (61\%), equivocal in one and completely negative in 13 . In the other 18 patients presenting mostly with aggressive or poorly differentiated tumours, FDG showed pathological localisation in $15(78 \%)$. These results are strictly associated with Tg levels, 14 patients with papillary thyroid carcinoma being in group I, 11 in group II and only eight in group III.

\section{Patient management}

The nPET-FDG findings led to a change in the management in seven patients (surgical treatment was decided or modified in patients nos $2,27,32,44$ and 45 , radiotherapy in patient no. 46 and redifferentiation therapy with retinoic acid analogues in patient no. 38).

The 12 patients with persistent elevated Tg levels (range $1.4-850 \mu \mathrm{g} / \mathrm{ml}$, mean $110.8 \mu \mathrm{g} / \mathrm{ml}$ ) and negative or equivocal imaging findings were carefully followed-up for 26 months (range 11-42) and at the time of writing remained without detectable metastases.

\section{Discussion}

The management of patients with recurrent differentiated thyroid cancer who cannot benefit from ${ }^{131} \mathrm{I}$ therapy is difficult. Possible therapeutic strategies include surgery, radiation therapy, chemotherapy and redifferentiation therapy with retinoic acid analogues, depending on the metastatic spread (22). Nowadays,

Table 4 Scintigraphic sensitivity according to histology and stage.

\begin{tabular}{|c|c|c|c|c|c|c|c|c|c|}
\hline \multirow[b]{2}{*}{ nPET } & \multicolumn{4}{|c|}{ Histology } & \multicolumn{5}{|c|}{ Stage } \\
\hline & $P$ & $\mathrm{~F}$ & 1 & $\mathrm{O}$ & I & II & III & IV & $x$ \\
\hline Positive & 20 & 9 & 3 & 2 & 6 & 17 & 7 & 2 & 2 \\
\hline Total & 33 & 11 & 4 & 3 & 13 & 19 & 10 & 2 & $\overline{7}$ \\
\hline Positive rate (\%) & 61 & \multicolumn{3}{|c|}{$78(\mathrm{~F}+\mathrm{I}+\mathrm{O})$} & \multicolumn{2}{|c|}{$55(\mathrm{I}+\mathrm{II})$} & \multicolumn{2}{|c|}{75 (III+IV) } & - \\
\hline Equivocal and 'all negative' patients & 7 & 2 & 1 & 0 & 4 & 2 & 1 & 0 & 5 \\
\hline
\end{tabular}

Histology: P, papillary; F, follicular; I, insular; and O, oncocytic thyroid carcinoma. 
only surgery is curative and it is crucial to detect early and localise accurately the recurrence. Since the identification of the tumour masses is often questionable with conventional imaging procedures, several scintigraphic methods have been proposed.

Enhanced glucose metabolism is a non-specific feature of tumour proliferative activity and fast-growing tumours are detectable with a high sensitivity by PET using FDG. The high sensitivity of PET-FDG in the detection of thyroid cancer has been demonstrated in a number of prospective studies (10, 12-14, 16-18). Glucose metabolism is generally increased in poorly differentiated carcinomas and FDG uptake correlates with malignant grade. An over-expression of the glucose transporter GLUT 1 was found in thyroid cancer with the most aggressive behaviour (15). Therefore, the discordance between radioiodine and FDG tumour uptake may reflect differences in functional differentiation. The intensity of FDG uptake could reflect tumour dedifferentiation and PET-FDG has been suggested as a prognostic indicator in thyroid cancers $(16-18)$.

In our study, all patients presented with elevated $\mathrm{Tg}$ levels and negative WBS. Apparently, in these patients, thyroid cancer tissue has lost the ability to take up iodine, but was still able to produce Tg. It is now well known that the mechanisms of iodine storage implies the sodium iodide symporter and that the synthesis of $\mathrm{Tg}$ is maintained longer in the process of dedifferentiation than is the ability to take up radioiodine (23). Thyroglobulin determination by a reference method (IRMA) is highly sensitive and specific (respectively $92 \%$ and $93 \%$ ) when obtained after withdrawal of thyroid hormone therapy, and in the absence of anti-Tg antibody. Persistent or recurrent disease was consequently assumed in all our patients, despite the fact that no lesion could be detected with any of the available methods in 10 cases. Thus, no false negative patient was possible and we could only assess the sensitivity of the imaging methods.

CIP were performed within 3 months of scintigraphic examinations, allowing comparison of the techniques for the identifications of lesions. While histopathological investigations of lesions in the neck area were obtained in all patients, this was not feasible for lung masses. We therefore admitted that the clinical follow-up and a congruence of CIP and nPET-FDG findings should represent confirmed disease.

In these conditions, the ability of nPET-FDG seems to vary with anatomical location. Cervical and mediastinal metastases are well detected by nPET-FDG whose diagnostic accuracy was similar or superior to that of CIP. Even if neck ultrasound resolution is typically $2-3 \mathrm{~mm}$, in some cases only the repetition of this exam with the awareness of nPET-FDG findings allowed the detection of a lesion. In five patients the lesion was surgically removed and histopathologically assumed as false negative for CIP and true positive for nPET-FDG. In our series, in fact, no false positive focus was detected by nPET-FDG in the neck region due to physiological muscle uptake or inflammatory uptake.

Conversely, false negatives of nPET were mostly small lung metastases, demonstrated by spiral CT, in agreement with previous reports $(14,19)$. Bone lesions were detected by nPET-FDG with a higher sensitivity than bone scintigraphy. In three patients, however, bone FDG uptake could not be fully interpreted since no biopsy was performed. It is well known that the sensitivity of bone scintigraphy in patient with thyroid cancer varies from 65 to $85 \%$, due to the presence of purely osteolytic lesions (24). The higher sensitivity of nPET may have been caused by differences in tracer uptake mechanism or different acquisition methods.

In the near future, fused images (simultaneous multimodality display of images) will allow anatomical localisation of uptake foci, thus improving sensitivity of both modalities and anatomical details of FDG examination.

In the present study, the influence of various factors on the sensitivity of nPET-FDG could be evaluated. Firstly the fraction of positive nPET-FDG increased with increasing $\mathrm{Tg}$ level, and this in accordance with the correlation between $\mathrm{Tg}$ level and the tumour mass. We found a significantly higher sensitivity in patients with $\mathrm{Tg}$ levels $>15 \mu \mathrm{g} / \mathrm{l}$, in accordance with previous studies (25). The rate of positive nPET-FDG was markedly lower in patients with papillary thyroid cancer, who may benefit more from neck ultrasound for the detection of small lymph nodes metastases. Conversely, the effects of TSH levels on the FDG uptake could not be established in our patients since nearly all scintigraphic examinations were performed during thyroid hormone suppressive therapy.

It is important to note that in our study, examinations performed with a dedicated PET were negative in all the patients showing no pathological FDG uptake on nPET. Therefore, on a patient basis, both methods showed the same efficacy. However, PET detected more lesions in one FDG-positive patient, without change in the therapeutic management.

We can therefore conclude that, before planning neck surgery, PET-FDG is useful for exploring the mediastinal region, mainly when CIP is inconclusive. A spiral CT of the lung is more efficient than FDG examination to exclude small pulmonary metastases. When ultrasound of the neck is inconclusive, or in patients with poorly differentiated thyroid carcinoma, and especially when an aggressive treatment is planned (i.e. before extensive surgery or external radiation therapy) PET-FDG is indicated. A possible routine algorithm is indicated in Table 5.

Finally, the modification of patient staging may suggest or contraindicate a therapy. After nPET-FDG, the clinical management changed in seven of our 51 patients. In particular, the precise localisation of the $\mathrm{Tg}$ production site allowed it to be subsequently totally 

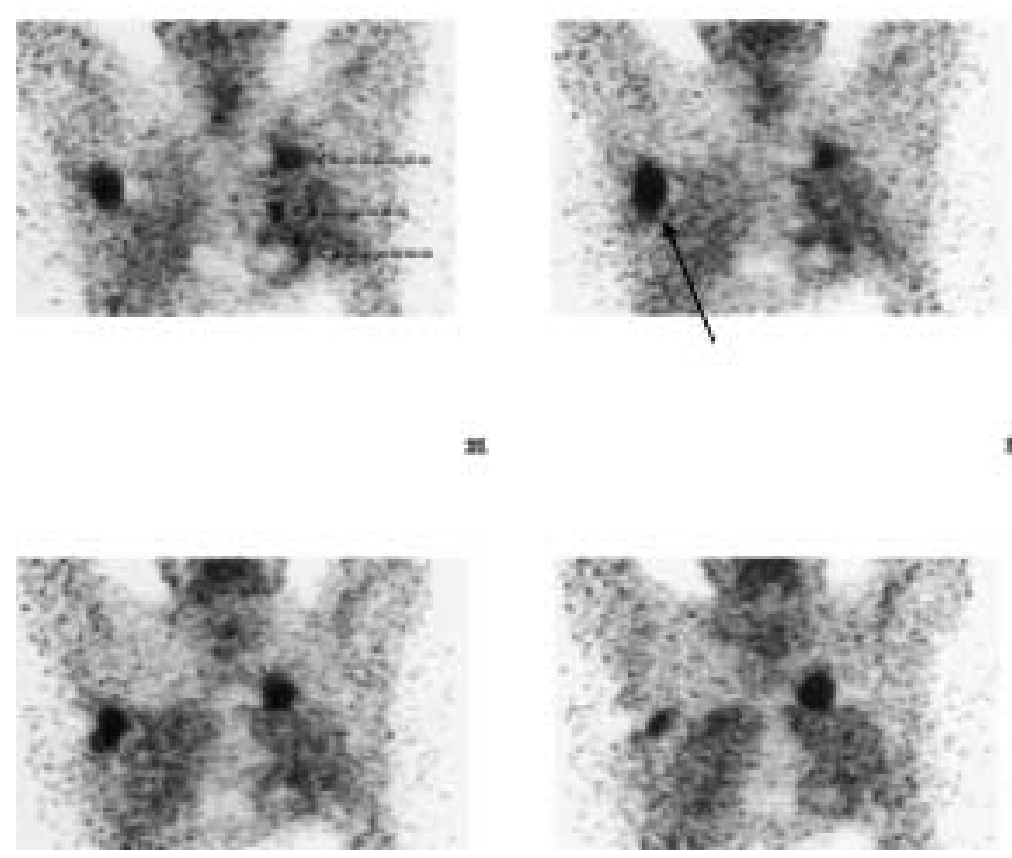

Figure $1 \mathrm{nPET}-F D G$ coronal views performed in patient no. 50, with inconclusive WBS and elevated Tg level $(283 \mu \mathrm{g} / \mathrm{ml}$ at the time of the examination, with TSH at $72 \mathrm{mU} / \mathrm{ml}$ ). Images (obtained $60 \mathrm{~min}$ after intravenous administration of $2 \mathrm{MBq} / \mathrm{kg}$ of FDG using a threehead large field-of-view gamma camera equipped with coincidence detection) showed multiple pathological increased uptakes in thoracic areas corresponding to unequivocal pulmonary (white arrows) and suspected bone (dark arrow) metastases. A CT scan confirmed the lung involvement while bone scan was normal.

Table 5 Routine diagnostic algorithm.

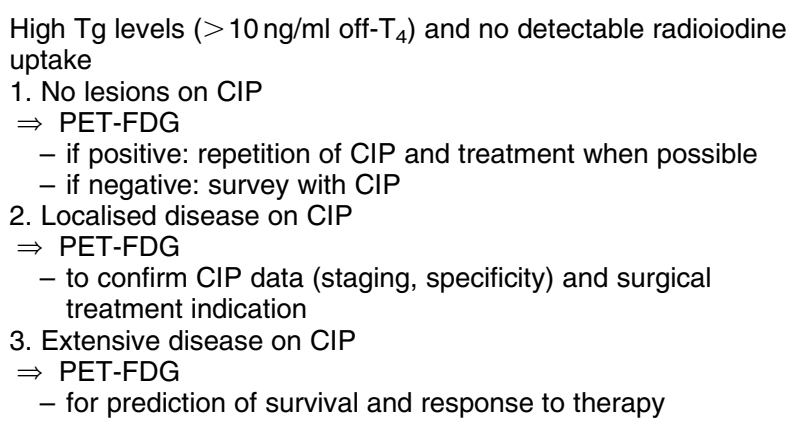

surgically removed in five patients. However, the clinical impact of the early recognition of a recurrent or metastatic thyroid cancer by means of PET-FDG in patients with persistent $\mathrm{Tg}$ levels and inconclusive WBS is still not demonstrated.

\section{Conclusion}

Our findings seem to support the diagnostic potential of nPET-FDG in patients with differentiated thyroid cancer who present with thyroglobulinaemia, mostly when
Tg levels are above $15 \mu \mathrm{g} / \mathrm{l}$, and when WBS, performed with a high dose of ${ }^{131} \mathrm{I}$, is inconclusive.

Although the clinical consequences of these observations have not been precisely documented, we suggest that PET using FDG could be used with high efficacy for the follow-up of patients with inconclusive WBS and elevated Tg levels.

\section{Acknowledgements}

This study was supported by a grant from the Hospices Civils de Lyon (PHRC 1997 and 2000). The authors thank Dr Véronique Lapras and Dr Philippe Thiesse for accurate radiological explorations and advice.

\section{References}

1 Pineda JD, Lee T, Ain K, Reynolds JC \& Robbins J. Iodine-131 therapy for thyroid cancer patients with elevated thyroglobulin and negative diagnostic scan. Journal of Clinical Endocrinology and Metabolism 199580 1488-1492.

2 Baudin E, Schlumberger M, Lumbroso J, Travagli JP, Caillou B \& Parmentier C. Octreotide scintigraphy in patients with differentiated thyroid carcinoma: contribution for patients with negative radioiodine scan. Journal of Clinical Endocrinology and Metabolism $1996812541-2544$. 
3 Schlumberger MJ. Papillary and follicular thyroid carcinoma. New England Journal of Medicine 1998338 297-306.

4 Alnafisi NS, Driedger AA, Coates G, Moote DJ \& Raphael SJ. FDG PET of recurrent or metastatic 131I-negative papillary thyroid carcinoma. Journal of Nuclear Medicine 200041 1010-1015.

5 Burman KD, Anderson JH, Wartofsky L, Mong DP \& Jelinek JJ. Management of patients with thyroid carcinoma: application of thallium-201 scintigraphy and magnetic resonance imaging. Journal of Nuclear Medicine 199031 1958-1964.

6 Dadparvar S, Chevres A, Tulchinsky M, Krishna-Badrinath L, Khan AS \& Slizofski WJ. Clinical utility of technetium-99m methoxisobutylisonitrile imaging in differentiated thyroid carcinoma: comparison with thallium-201 and iodine-131 Na scintigraphy, and serum thyroglobulin quantitation. European Journal of Nuclear Medicine 199522 1330-1338.

7 Valli N, Catargi B, Ronci N, Leccia F, Guyot M, Roger P et al. Evaluation of indium-111 pentetreotide somatostatin receptor scintigraphy to detect recurrent thyroid carcinoma in patients with negative radioiodine scintigraphy. Thyroid 1999 9 583-589.

8 Garin E, Devillers A, Le Cloirec J, Bernard AM, Lescouarc'h J, Herry JY et al. Use of indium-111 pentetreotide somatostatin receptor scintigraphy to detect recurrent thyroid carcinoma in patients without detectable iodine uptake. European Journal of Nuclear Medicine 199825 687-694.

9 Strauss LG \& Conti PS. The applications of PET in clinical oncology. Journal of Nuclear Medicine 199132 623-648.

10 Grunwald F, Kalicke T, Feine U, Lietzenmayer R, Scheidhauer K, Dietlein $\mathrm{M}$ et al. Fluorine-18 fluorodeoxyglucose positron emission tomography in thyroid cancer: results of a multicentre study. European Journal of Nuclear Medicine 199926 1547-1552.

11 Helal BO, Merlet P, Toubert ME, Franc B, Schvartz C, Gauthier-Koelesnikov H et al. Clinical impact of (18)F-FDG PET in thyroid carcinoma patients with elevated thyroglobulin levels and negative (131)I scanning results after therapy. Journal of Nuclear Medicine 200142 1464-1469.

12 Sisson JC, Ackermann RJ, Meyer MA \& Wahl RL. Uptake of 18-fluoro-2-deoxy-D-glucose by thyroid cancer: implications for diagnosis and therapy. Journal of Clinical Endocrinology and Metabolism 199377 1090-1094.

13 Feine U, Lietzenmayer R, Hanke JP, Held J, Wohrle $\mathrm{H}$ \& Muller-Schauenburg W. Fluorine-18-FDG and iodine-131-iodide uptake in thyroid cancer. Journal of Nuclear Medicine 199637 $1468-1472$

14 Dietlein M, Scheidhauer K, Voth E, Theissen P \& Schicha H. Fluorine-18 fluorodeoxyglucose positron emission tomography and iodine-131 whole-body scintigraphy in the follow-up of differentiated thyroid cancer. European Journal of Nuclear Medicine 1997 24 1342-1348.

15 Schonberger J, Ruschoff J, Grimm D, Marienhagen J, Rummele P, Meyringer R et al. Glucose transporter 1 gene expression is related to thyroid neoplasms 747 with an unfavorable prognosis: an immunohistochemical study. Thyroid 200212 747-752.

16 Chung JK, So Y, Lee JS, Choi CW, Lim SM, Lee DS et al. Value of FDG PET in papillary thyroid carcinoma with negative 131I whole-body scan. Journal of Nuclear Medicine 1999 40 986-992.

17 Wang PW, Wang ST, Liu RT, Chien WY, Tung SC, Lu YC et al. Levothyroxine suppression of thyroglobulin in patients with differentiated thyroid carcinoma. Journal of Clinical Endocrinology and Metabolism $1999 \mathbf{8 4} 4549-4553$.

18 Wang W, Larson SM, Fazzari M, Tickoo SK, Kolbert K, Sgouros G et al. Prognostic value of [18F]fluorodeoxyglucose positron emission tomographic scanning in patients with thyroid cancer. Journal of Clinical Endocrinology and Metabolism 2000 85 1107-1113.

19 Stokkel MP, de Klerk JH, Zelissen PM, Koppeschaar HP \& van Rijk PP. Fluorine-18 fluorodeoxyglucose dual-head positron emission tomography in the detection of recurrent differentiated thyroid cancer: preliminary results. European Journal of Nuclear Medicine 199926 1606-1609.

20 Tiepolt C, Beuthien-Baumann B, Hliscs R, Bredow J, Kuhne A, Kropp J et al. 18F-FDG for the staging of patients with differentiated thyroid cancer: comparison of a dual-head coincidence gamma camera with dedicated PET. Annals of Nuclear Medicine $200014339-345$

21 Sherman SI, Brierley JD, Sperling M, Ain KB, Bigos ST, Cooper DS et al. Prospective multicenter study of thyroid carcinoma treatment: initial analysis of staging and outcome. National Thyroid Cancer Treatment Cooperative Study Registry Group. Cancer $1998831012-1021$.

22 Lerch H, Schober O, Kuwert $\mathrm{T}$ \& Saur HB. Survival of differentiated thyroid carcinoma studied in 500 patients. Journal of Clinical Oncology 199715 2067-2075.

23 Spitzweg C, Harrington KJ, Pinke LA, Vile RG \& Morris JC. The sodium iodide symporter and its potential role in cancer therapy. Journal of Clinical Endocrinology and Metabolism $2001 \mathbf{8 6}$ $3327-3335$.

24 Schirrmeister H, Buck A, Guhlmann A \& Reske SN. Anatomical distribution and sclerotic activity of bone metastases from thyroid cancer assessed with F-18 sodium fluoride positron emission tomography. Thyroid 2001 $11677-683$.

25 Schluter B, Bohuslavizki KH, Beyer W, Plotkin M, Buchert R \& Clausen M. Impact of FDG PET on patients with differentiated thyroid cancer who present with elevated thyroglobulin and negative 131I scan. Journal of Nuclear Medicine 2001 42 71-76.

Received 2 May 2003

Accepted 10 July 2003 